\title{
Evaluación breve de la presencia de diatomeas y su relación con algunos parámetros físico-químicos en el río Pirro, Heredia, Costa Rica
}

\author{
Brief Assessment of the Presence of Diatoms and Their Relationship with Some \\ Physical and Chemical Parameters in the Pirro River, Heredia, Costa Rica
}

\author{
Eunice Flores-Stulzer \\ eunice fsp@hotmail.com \\ Escuela de Ciencias Biológicas \\ Universidad Nacional \\ Heredia, Costa Rica \\ Narcy Villalobos-Sandí \\ narcy.villalobos.sandi@una.cr \\ Escuela de Ciencias Biológicas \\ Universidad Nacional \\ Heredia, Costa Rica \\ Lilliana Piedra-Castro \\ lilliana.piedra.castro@una.cr \\ Escuela de Ciencias Biológicas \\ Universidad Nacional \\ Heredia, Costa Rica \\ Carola Scholz \\ carola.scholz@una.cr \\ Escuela de Ciencias Biológicas \\ Universidad Nacional \\ Heredia, Costa Rica
}

Recibido-Received: 13/dic/2015 / Corregido-Corrected: 5/set /2016.

Aceptado-Accepted: 23/set/2016 / Publicado-Published: 31/jul /2017.

\begin{abstract}
Resumen
La evaluación de la calidad de agua en ecosistemas fluviales en áreas urbanas en el trópico, basada en la utilización de diatomeas, es escasa. Este estudio exploratorio pretendió establecer la composición y riqueza de especies de diatomeas en relación con los parámetros físicos químicos como elemento base para un monitoreo de la microcuenca urbana del río Pirro, Heredia, Costa Rica. Los materiales se colectaron en la parte alta, media y baja del río durante la época seca, lluviosa y de transición. Se analizó el pH, el oxígeno disuelto, la conductividad, la temperatura y la composición de diatomeas
\end{abstract}


UNICIENCIA Vol. 31, No. 2, pp.99-109. Julio-diciembre, 2017.

ISSN Electrónico: 2215-3470

URL: www.revistas.una.ac.cr/uniciencia

DOI: http://dx.doi.org/10.15359/ru.31-2.7

Email: revistauniciencia@una.cr

raspando una superficie de $20 \mathrm{~cm} \times 20 \mathrm{~cm}$ de rocas. Se observaron 18 especies de diatomeas con poca abundancia, con presencia de Navicula seminulum, N. minima y N. cryptotenella que son indicadoras de condiciones moderadas de contaminación y del género Nitzschia que presenta especies tolerantes a contaminantes. La mayor diversidad de especies de diatomeas ocurrió en la época lluviosa y se observaron diferencias significativas entre las épocas del muestreo y la riqueza de especies. Se concluye, con base en la presencia de especies, que el río Pirro presenta condiciones sanitarias malas y que el cauce bajo es el sitio más afectado por la contaminación. Pero es necesario aumentar la información en esta temática.

Palabras claves: Costa Rica; zona urbana; riqueza; composición.

\begin{abstract}
The assessment of water quality in river ecosystems located in urban areas in the tropics based on the use of diatoms is scarce. This exploratory study aimed to establish the composition and species richness of diatoms in relation to physicochemical parameters as base element for monitoring the Pirro urban micro-watershed river in Heredia, Costa Rica. Materials were collected in the upper, middle, and lower river during dry, rainy, and transitional seasons. Dissolved oxygen, $\mathrm{pH}$, conductivity, temperature, and composition diatomaceous were evaluated by scraping an area of 20 $\mathrm{cm}$ x $20 \mathrm{~cm}$ rocks. 18 diatom species with low abundance such as Navicula seminulum, N. minimum and N. cryptotenella were observed. These are indicatives of moderate conditions of pollution; others kinds, such as Nitzschia, display tolerant species to contaminants. The greatest diversity of species of diatoms occurred in the rainy season, and significant differences between sampling season and species richness were observed. In conclusion, Pirro river has poor sanitary conditions based on the presence of many different species, and the low channel of the river is the most affected by pollution. However, it is necessary to boost the information on this subject.
\end{abstract}

Keywords: Costa Rica; Urban areas; Wealth; Composition

Los ecosistemas en todo el mundo han estado influenciados directa e indirectamente por las actividades humanas, y han provocado graves problemas, como la contaminación de los ambientes acuáticos y las aguas subterráneas, la deforestación y la introducción de especies exóticas, entre otros (Goulart \& Callisto, 2003).

En el caso particular de Costa Rica, el recurso agua sufre una gran crisis, debido al crecimiento descontrolado y sin planificación de zonas urbanizadas, sobre todo en la Gran Área Metropolitana; esto ha generado un aumento de la contaminación de los acuíferos y de los ríos en esta región, aumento de las tendencias migratorias del campo a la ciudad y la invasión de las zonas de protección con infraestructura (Ballestero, 2003). Todo lo anteriormente citado se suma a un complejo y desarticulado marco jurídico-institucional con pocos mecanismos de evaluación de la calidad de agua para una gestión eficaz y de prevención en la conservación de los recursos hídricos.

Dentro de estos, especialmente los ecosistemas rivereños han sufrido un gran daño, debido a la rápida extensión de urbanizaciones o la urbanización incontrolada. Durante muchos años, las aguas residuales de origen doméstico e industrial han finalizado en los cuerpos de agua haciendo que estos pierdan su función y, por ende, lleven al deterioro también del ecosistema urbano. Por esta razón urge el desarrollo de técnica efectivas de bajo costo que permitan la clasificación de la calidad del agua de los ríos para planificar su manejo. 
Una forma utilizada para la evaluación de las condiciones de un sistema acuático ha sido el uso de parámetros físico-químicos del agua; sin embargo, dichos parámetros solo muestran las condiciones establecidas en un momento determinado (Fegnolio et al., 2002). Por tanto, el uso de bioindicadores para el análisis de la calidad del agua ha venido tomando fuerza a través de los años, constituye un diagnóstico de los cambios que han ocurrido a través del tiempo, lo cual permite

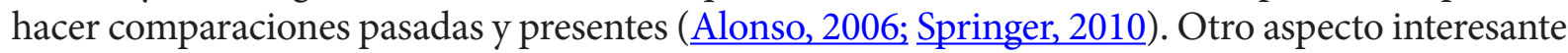
con la utilización de indicadores biológicos es la detección de eventos puntuales de toxicidad, lo cual, por lo general, no es posible medir con parámetros físico-químicos (Springer, 2010).

En Costa Rica, por su parte, el uso de bioindicadores de contaminación es un campo que hasta hace pocos años comienza a tener fuerza. Con el fin de reglamentar los criterios y metodologías que se utilizan para la evaluación de la calidad de los cuerpos de agua superficiales y permitir su clasificación para los diferentes usos que se le pueda dar, se desarrolló el "Reglamento para la evaluación y clasificación de la calidad de cuerpos de agua superficiales", publicado en La Gaceta en setiembre del 2007 (MINAE-S No. 33903).

En este reglamento se contempla, además, por primera vez, el uso de bioindicadores; específicamente el uso de macroinvertebrados en adición al método físico-químico para la determinación de la calidad del agua.

Por otro lado, en países europeos se ha implementado la utilización de microalgas, sobre todo del grupo de las diatomeas como bioindicadoras de la calidad de los cuerpos de agua, desde los años setenta (Blanco et al., 2007). Lo anterior ha resultado en la creación de varios índices que se pueden utilizar para medir el grado de alteración de los ríos (Penalta-Rodríguez \& López-Rodríguez, 2007). De acuerdo con Ector y Rimet (2005, citado por Blanco et al., 2007), los índices más utilizados en Europa son el índice de polusensibilidad específica (IPS), el índice CEE; el índice biológico de diatomeas (IBD), los índices sapróbico SI y trófico TI de Rott, el índice de eutrofización/polución de Dell'Uomo y el índice trófico de diatomeas TDI.

A pesar de contar con bastante información de otros países, el estudio, análisis e implementación de índices diatomológicos para Costa Rica es escaso. Aunque se conoce su utilidad, es necesario contar con una base florística de diatomeas que sirva de referencia para generar conclusiones (Siqueiros-Beltrones et al., 2014).

El propósito de este trabajo fue establecer la composición y riqueza de especies de diatomeas (Bacillariophyceae) en relación con los parámetros físicos y químicos como elemento base para futuros monitoreos que contribuyan con el manejo de esta microcuenca urbana y de otros cuerpos de agua del país.

\section{Metodología}

\section{Descripción del sitio de estudio}

Este estudio se llevó a cabo en la microcuenca del río Pirro, afluente del río Bermúdez en la ciudad de Heredia, Costa Rica. Esta cuenca posee una extensión de 7,3 km², con una elevación máxima de 1420 m.s.n.m y una mínima de 1050 m.s.n.m. Recorre los cantones de San Rafael, central de Heredia y San Pablo (Romero, 2008).

La temperatura promedio anual es de $20,1^{\circ} \mathrm{C}$, la humedad relativa promedio de $79 \%$, con una precipitación promedio anual de 2374,3 mm. Según Miranda et al. (2010), el uso del suelo en 
UNICIENCIA Vol. 31, No. 2, pp.99-109. Julio-diciembre, 2017.

ISSN Electrónico: 2215-3470

URL: www.revistas.una.ac.cr/uniciencia

DOI: http://dx.doi.org/10.15359/ru.31-2.7

Email: revistauniciencia@una.cr

esta microcuenca está caracterizado por urbanismo (70\%), cafetales (18\%), pastos y vegetación ( $6 \%$ cada uno), esto permite afirmar que la microcuenca del río Pirro es típicamente urbana.

$\mathrm{Su}$ cauce principal presenta obras de entubamiento desde su naciente y, además, está catalogado como un cauce intermitente, lo que quiere decir que únicamente en época lluviosa presenta caudal, sin embargo, debido al vertedero de aguas servidas, este río presenta un caudal permanente (Romero et al., 2011).

\section{Muestreo}

Se establecieron tres puntos de muestreo, el primero localizado en San Rafael de Heredia (cauce alto del río), el segundo ubicado en el campus Omar Dengo de la Universidad Nacional (cauce medio del río) y el último, en el barrio Guayabal de Heredia (cauce bajo del río). En cada sitio se realizaron tres muestreos, uno durante la estación lluviosa, otro en la estación seca y el último en la época de transición entre la estación seca y lluviosa. Durante cada muestreo se midió directamente en el agua el $\mathrm{pH}$, el oxígeno disuelto, la conductividad y la temperatura con un multiparámetro Thermo Scientific Orion 5 Star.

La recolección de diatomeas se realizó raspando una superficie de $20 \mathrm{~cm} \mathrm{x} 20 \mathrm{~cm}$ de 3 rocas seleccionadas al azar en el centro del cauce del río y en ambas orillas. Las piedras fueron raspadas con un cepillo de dientes, el cual fue lavado con agua del mismo punto y colocado en un recipiente extendido. A partir de ahí, se colectó una muestra viva en botellas de plástico de 1.5 litros (Rice et al., 2012) y se trasladaron al laboratorio de Biotecnología de Microalgas, de la Escuela de Ciencias Biológicas de la Universidad Nacional para su preparación e identificación.

\section{Preparación de las muestras}

Para realizar la descripción de las diatomeas se realizó una digestión de la muestra, en donde se tomaron $2 \mathrm{ml}$ de esta misma, se añadieron $2 \mathrm{ml}$ de peróxido de hidrogeno al 10\% y se dejaron reposar en tubos de ensayo por 17 horas (para digerir la mayor cantidad de materia orgánica) (Silva-Benavidez et al., 2008).

Posteriormente, se realizó una sedimentación de la muestra por centrifugación a 1500 rpm por 5 min y luego se procedió al lavado. Se retiró el peróxido de hidrógeno, se realizaron lavados con agua destilada y, por último, se centrifugó de nuevo a la misma velocidad durante $2 \mathrm{~min}$. De cada muestra se elaboraron preparaciones microscópicas con resina sintética (Naphrax ${ }^{\circledR}$ ) y se observaron bajo el microscopio de luz marca Olympus $\mathrm{CH}$.

\section{Análisis estadístico}

El análisis de los datos de los parámetros físico químicos y la riqueza de especies de diatomeas en los sitios de muestreo consistió en las pruebas de normalidad, análisis de varianza (ANOVA) y la comparación de medias de Tukey, así como también se aplicó el índice de disimilitud de Sorensen. Dichos análisis fueron realizados en el programa estadístico InfoStat (versión 2013I).

\section{Resultados y discusión}

Los sitios de muestreo presentaron características similares en cuanto a la apariencia de sus aguas, vegetación y algunos problemas (tabla 1). En general, durante todos los muestreos, el agua se presentó turbia, con fuertes olores, mucha basura y deslizamientos de los taludes y laderas. 
ISSN Electrónico: 2215-3470

DOI: http://dx.doi.org/10.15359/ru.31-2.7
UNICIENCIA Vol. 31, No. 2, pp. 99-109. Julio-diciembre, 2017.

URL: www.revistas.una.ac.cr/uniciencia Email: revistauniciencia@una.cr

Tabla 1

Características generales de los sitios de muestreo, río Pirro, Heredia Costa Rica

\begin{tabular}{clll}
\hline Sitio de muestreo & Ubicación & Localización & Características del sitio \\
\hline 1 & Cauce alto & $\begin{array}{l}\text { San Rafael de } \\
\text { Heredia }\end{array}$ & $\begin{array}{l}\text { En este sitio, una parte del río se encuentra entubada, el agua es } \\
\text { bastante turbia, el cauce está muy cerca de las viviendas, con un } \\
\text { espejo de agua muy angosto (1,5 m promedio). }\end{array}$ \\
\hline 2 & Cauce medio & $\begin{array}{l}\text { Campus } \\
\text { Omar Dengo, } \\
\text { UNA }\end{array}$ & $\begin{array}{l}\text { El cauce es más ancho y profundo, con presencia de pozas } \\
\text { problemas de deslizamientos y aguas grises y negras. }\end{array}$ \\
\hline \multirow{2}{*}{3} & Cauce bajo & $\begin{array}{l}\text { Guayabal de } \\
\text { Heredia }\end{array}$ & $\begin{array}{l}\text { En este sitio el cauce presentó más corriente de agua; pero } \\
\text { se encuentra rodeado de gran cantidad de infraestructura y } \\
\text { poca vegetación. Es más profundo con muchos problemas de } \\
\text { deslizamientos. Presencia de aguas jabonosas ybastante basura. }\end{array}$ \\
& & &
\end{tabular}

Nota: Elaboración propia.

En cuanto a las mediciones físico-químicas, los sitios de muestreo presentaron valores de $\mathrm{pH}$ ligeramente que oscilan entre 7.00 y 7.44, con excepción de la época de transición en donde hubo una variación en la cuenca alta y baja (5.90 y 6.50 respectivamente). Los valores de oxígeno disuelto (3.19-6.91 mg/L) son bajos y la conductividad muestra valores variables $(172-345 \mathrm{um} / \mathrm{cm})($ tabla 2$)$.

Tabla 2

Mediciones ambientales de los sitios de muestreo, río Pirro Heredia, Costa Rica

\begin{tabular}{ccccc}
\hline Época & Sitios & $\mathrm{pH}$ & OD $(\mathrm{mg} / \mathrm{L})$ & Conductividad $(\mathrm{uS} / \mathrm{cm})$ \\
\hline Lluviosa & Alta & 7,13 & 3,82 & 345 \\
& Media & 7,29 & 5,40 & 206 \\
& Baja & 7,03 & 4,60 & 190 \\
Seca & Alta & 7,30 & 6.91 & 179 \\
& Media & 7,42 & 5,93 & 209 \\
& Baja & 7,44 & 5,89 & 172 \\
Transición & Alta & 5,90 & 0,98 & 316 \\
& Media & 7,00 & 3,19 & 327 \\
& Baja & 6,50 & 3,88 & 331 \\
\hline
\end{tabular}

Nota: Elaboración propia. 
UNICIENCIA Vol. 31, No. 2, pp.99-109. Julio-diciembre, 2017.

Los datos anteriores indican que este río presenta condiciones muy inestables con parámetros ambientales que cambian a lo largo del año. Estos cambios pueden deberse a las condiciones características propias de cada época, las cuales hacen que las sustancias orgánicas y otros contaminantes, que son depositados en el río, se encuentren más diluidos en época lluviosa y más concentrados en la época seca, tal es el caso del oxígeno disuelto que mostró un incremento en la época seca, posiblemente debido a que había menos cantidad de agua.

En la microcuenca se encontró un total de 18 especies de diatomeas, con poca abundancia de individuos (tabla 3). Algunas de estos géneros o sus especies ya han sido reportadas para Costa Rica en otros estudios con algunas consideraciones sobre la información que puedan brindar en cuanto a indicadora de contaminación (Silva-Benavides et al., 2008).

Por otro lado, se considera que cuanto mayor es la diversidad y abundancia de especies existentes en el medio, las aguas son de mejor calidad (Sabater, Sabater y Tomas, 1987); en este sentido, la diversidad de diatomeas encontrada es baja, por lo que es un indicativo de la mala calidad en que se encuentran las aguas del río Pirro en los sectores muestreados.

Tabla 3

Especies de diatomeas observadas en el río Pirro, Heredia, Costa Rica

\begin{tabular}{|c|c|c|c|c|c|c|c|c|c|}
\hline Época & \multicolumn{3}{|c|}{ Lluviosa } & \multicolumn{3}{|c|}{ Seca } & \multicolumn{3}{|c|}{ Transición } \\
\hline Especie & San Rafael & UNA & Guararí & $\begin{array}{c}\text { San } \\
\text { Rafael }\end{array}$ & UNA & Guararí & $\begin{array}{c}\text { San } \\
\text { Rafael }\end{array}$ & UNA & Guararí \\
\hline
\end{tabular}

Achnanthidium

minutissimum

(Kützing, Czarnecki

1994)

Amphora normanii

(Rabenhorst 1864)

Frustulia vulgaris

(Thwaites, De Toni

1891)

Gomphonema parvalum

(Kützing 1849)

Hantzschia amphioxys

(Ehrenberg, Grunow in

Cleve \& Grunow 1880)

Navicula

capitatoradiata

(H.Germain 1981)

Navicula cryptotenella

(Lange-Bertalot 1985)

X $\quad \mathrm{X}$

X

X

X $\quad$ X $\quad$ X

$\mathrm{X}$ 
ISSN Electrónico: 2215-3470

DOI: http://dx.doi.org/10.15359/ru.31-2.7
UNICIENCIA Vol. 31, No. 2, pp. 99-109. Julio-diciembre, 2017.

URL: www.revistas.una.ac.cr/uniciencia Email: revistauniciencia@una.cr

\begin{tabular}{|c|c|c|c|c|c|c|c|c|c|}
\hline \multirow{2}{*}{$\frac{\text { Época }}{\text { Especie }}$} & \multicolumn{3}{|c|}{ Lluviosa } & \multicolumn{3}{|c|}{ Seca } & \multicolumn{3}{|c|}{ Transición } \\
\hline & San Rafael & UNA & Guararí & $\begin{array}{c}\text { San } \\
\text { Rafael }\end{array}$ & UNA & Guararí & $\begin{array}{c}\text { San } \\
\text { Rafael }\end{array}$ & UNA & Guararí \\
\hline $\begin{array}{l}\text { Navicula minima } \\
\text { (Grunow 1880) }\end{array}$ & $X$ & $X$ & $X$ & $X$ & & & $X$ & $X$ & $X$ \\
\hline $\begin{array}{l}\text { Navicula } \\
\text { protracta (Grunow, } \\
\text { Cleve 1894) }\end{array}$ & $\mathrm{X}$ & $\mathrm{X}$ & $\mathrm{X}$ & $\mathrm{X}$ & $\mathrm{X}$ & $\mathrm{X}$ & & & \\
\hline $\begin{array}{l}\text { Navicula sp. (Hassall } \\
\text { 1845) }\end{array}$ & $\mathrm{X}$ & $\mathrm{X}$ & $\mathrm{X}$ & $\mathrm{X}$ & & & $\mathrm{X}$ & $\mathrm{X}$ & $\mathrm{X}$ \\
\hline $\begin{array}{l}\text { Nitzschia amphibian } \\
\text { (Grunow, 1862) }\end{array}$ & $\mathrm{X}$ & $\mathrm{X}$ & $\mathrm{X}$ & & $\mathrm{X}$ & $\mathrm{X}$ & & & $\mathrm{X}$ \\
\hline Nitzschia filiformis & $\mathrm{X}$ & $\mathrm{X}$ & $\mathrm{X}$ & & & $\mathrm{X}$ & $\mathrm{X}$ & $\mathrm{X}$ & $\mathrm{X}$ \\
\hline $\begin{array}{l}\text { Nitzschia palea } \\
\text { (Kutzing, W Smith } \\
\text { 1856) }\end{array}$ & $\mathrm{X}$ & $\mathrm{X}$ & $\mathrm{X}$ & & $\mathrm{X}$ & $\mathrm{X}$ & $\mathrm{X}$ & $\mathrm{X}$ & $\mathrm{X}$ \\
\hline $\begin{array}{l}\text { Nitzschia recta } \\
\text { (Hantzsch ex } \\
\text { Rabenhorst 1862) }\end{array}$ & $\mathrm{X}$ & & $\mathrm{X}$ & & $\mathrm{X}$ & $\mathrm{X}$ & $\mathrm{X}$ & $\mathrm{X}$ & \\
\hline $\begin{array}{l}\text { Nitzschia sp. (Hassall } \\
18459\end{array}$ & $\mathrm{X}$ & $\mathrm{X}$ & $\mathrm{X}$ & $\mathrm{X}$ & $\mathrm{X}$ & $\mathrm{X}$ & $\mathrm{X}$ & $\mathrm{X}$ & $\mathrm{X}$ \\
\hline $\begin{array}{l}\text { Pinnularia sp. } \\
\text { (Ehrenberg 1943) }\end{array}$ & $\mathrm{X}$ & $\mathrm{X}$ & $\mathrm{X}$ & & $\mathrm{X}$ & $\mathrm{X}$ & $\mathrm{X}$ & $\mathrm{X}$ & $\mathrm{X}$ \\
\hline $\begin{array}{l}\text { Sellaphora seminulum } \\
\text { (Mann 1989) }\end{array}$ & $\mathrm{X}$ & $\mathrm{X}$ & $\mathrm{X}$ & $\mathrm{X}$ & $\mathrm{X}$ & $\mathrm{X}$ & $\mathrm{X}$ & $\mathrm{X}$ & $\mathrm{X}$ \\
\hline $\begin{array}{l}\text { Stauroneis } \\
\text { phoenicenteron. } \\
\text { (Ehrenberg 1941) }\end{array}$ & $\mathrm{X}$ & & & & & & & & \\
\hline Total & 16 & 11 & 15 & 7 & 9 & 11 & 9 & 9 & 9 \\
\hline
\end{tabular}

Nota: Elaboración propia.

En cuanto a las especies Navicula seminulum, N. minima y N. cryptotenella son indicadoras de condiciones moderadas de contaminación del agua (Kelly, 2000, Silva-Benavides et al., 2008). Además, el género Nitzschia presenta especies tolerantes a contaminantes con altos valores de nitrógeno, fosforo y carbono (Werner, 1977). En general, las especies de los géneros Nitzschia y Navicula es posible encontrarlas en lugares semiurbanos en donde la contaminación presente favorece su crecimiento (Kelly, 2000, Silva-Benavides et al., 2008). 
UNICIENCIA Vol. 31, No. 2, pp.99-109. Julio-diciembre, 2017.

De acuerdo con la información anterior, en el cauce alto del río fue posible observar mayor cantidad de especies, que en el cauce bajo. Sin embargo, no se encontraron diferencias significativas entre los puntos de muestreo (ANOVA, $\mathrm{p}=0.7703$ ). A pesar de esto, es de esperar que el cauce bajo sea un sitio más afectado por la contaminación generada en todo el trayecto. En este sentido, Michels (1998) reporta haber encontrado cambios en los porcentajes de ciertos grupos de especies de diatomeas relacionados con el incremento o decrecimiento de la contaminación en dos ríos de Costa Rica, por esta razón concluye que estos organismos son útiles como indicadores de la contaminación en cuerpos de agua.

Sumado a esto, se observa mayor diversidad de especies de diatomeas en la época lluviosa, en donde se pudieron verificar diferencias significativas entre las épocas del muestreo (figura 1) (ANOVA, $\mathrm{p}=0.0285$ ).

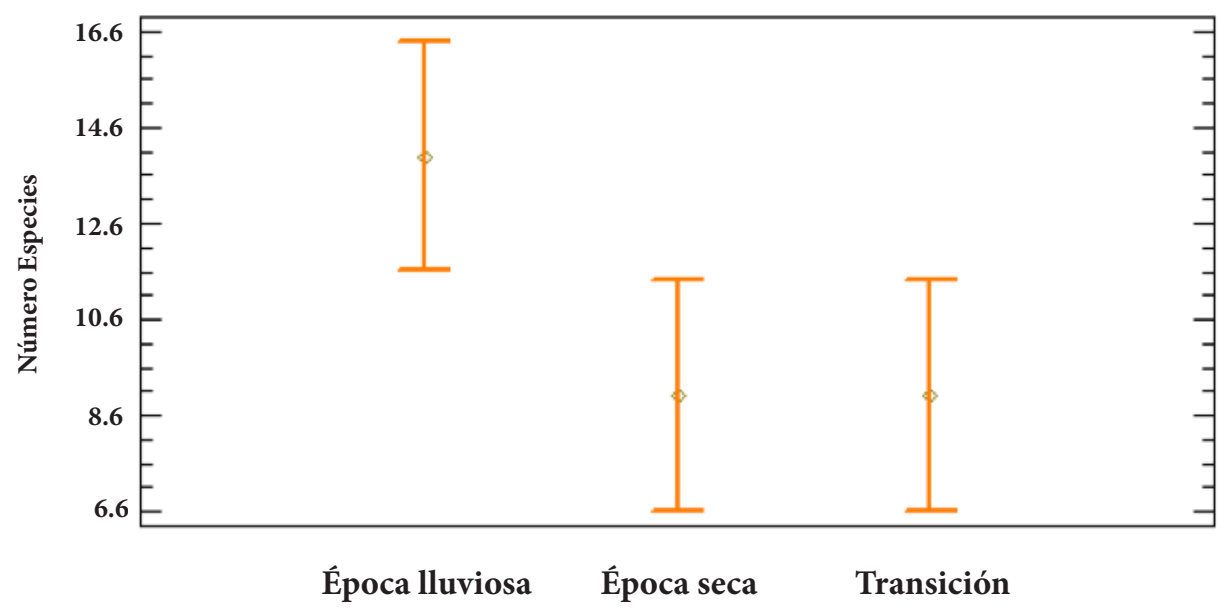

Figura 1. Diversidad de especies de diatomeas en tres estaciones de muestreo en épocas distintas río Pirro, Heredia, Costa Rica. Elaboración propia.

De acuerdo con la similitud de especies en los sitios por épocas de muestreo, se obtuvo que la composición de diatomeas en el sector de San Rafael presentó una variación de un $28 \%$ comparando la época lluviosa y seca, mientras que entre la época lluviosa y la de transición esta fue de $47.8 \%$, por último, entre la época seca y la de transición alcanzó un 50\%. En el caso del sector encontrado en el campus de la UNA, la variación se comportó de la siguiente forma: entre la época lluviosa y seca la similitud fue de $20 \%$, entre la época de transición y la lluviosa fue de $30 \%$ y entre la época seca y de transición fue de $33 \%$. En Guayabal la variación se dio en un $25 \%$ entre las épocas lluviosa y seca, un $36 \%$ entre la época de transición y la lluviosa y un $47 \%$ la época seca y de transición.

En el caso de similitudes entre sitios en la misma época de muestreo, se observa que en la época lluviosa y de transición los tres sitios presentaron mucha similitud. Sin embargo, en la época seca hubo gran variación entre Guayabal y la estación de la UNA con respecto a San Rafael (tabla 4). En el caso de la época lluviosa la composición de especies de diatomeas en los sectores de la UNA, Guayabal y San Rafael varió entre 15 a 18\%, donde son El Guayabal y la UNA los sectores con menor variación (15\%), mientras que en la época seca el porcentaje de disimilitud en estos sitios obtuvo los valores más altos al comparar la composición de especies de la UNA o Guayabal con el sector de San Rafael con un porcentaje de diferencia de 65 y 76\%, mientras que 
la UNA y Guayabal presentaron baja variación con un 15\%. Por último, la diferencia entre los sectores de Guayabal-San Rafael y Guayabal-UNA fue de 22\% y San Rafael-UNA de 11\%.

Tabla 4

Comparación de las especies de diatomeas encontradas entre los tres sitios de muestreo para cada época, río Pirro, Heredia, Costa Rica

\begin{tabular}{llc}
\hline Comparación por sector & Época & Disimilitud \\
\hline UNA-San Rafael & Lluviosa & $18 \%$ \\
Guayabal-San Rafael & Lluviosa & $16 \%$ \\
Guayabal-UNA & Lluviosa & $15 \%$ \\
UNA-San Rafael & Seca & $62 \%$ \\
Guayabal-San Rafael & Seca & $76 \%$ \\
Guayabal-UNA & Seca & $15 \%$ \\
UNA-San Rafael & Transición & $11 \%$ \\
Guayabal-San Rafael & Transición & $22 \%$ \\
Guayabal-UNA & Transición & $22 \%$ \\
\hline
\end{tabular}

Nota: Elaboración propia.

A pesar de contar con poca información ecológica de las diatomeas de ríos urbanos en Costa Rica, esta breve evaluación ofrece información de tipo exploratorio que permite continuar con monitoreos en otros ríos del país, que hoy se encuentran muy afectados por actividades relacionadas con el desarrollo humano. El uso de base de datos basadas en diatomeas más que en parámetros físico-químicos, para la clasificación de los ecosistemas acuáticos, está siendo empleado en algunos países de Europa para la toma de decisiones en el campo del manejo ambiental (Naumoski \& Mitreski, 2010).

Estas bases de datos proporcionan información que permite modelar el cuerpo de agua, por tanto, hacen más fácil la interpretación de los datos. En el caso de Costa Rica, este tipo de herramientas informáticas también podría ser utilizado en la medida en que se conozca más sobre este grupo de organismos, de ahí que sea tan importante su estudio.

Además, debido a la importancia ecológica que tienen las microalgas y la relevancia que han adquirido las diatomeas, específicamente, en el monitoreo de aguas superficiales en otros países, es necesario realizar más investigación en esta línea, con el fin de establecer los lineamientos necesarios para generar índices diatomológicos aplicables en el país, que puedan ser usados como complemento al índice de macroinvertebrados existente a nivel de regulación.

El río Pirro presenta condiciones sanitarias bastante deterioradas. Hay una variación entre los parámetros medidos en la parte alta del río con respecto a la parte baja; sin embargo, esta variación es característica de un ecosistema altamente contaminado con aguas vertidas que genera parámetros muy variados a lo largo del año. Por esta razón no existen diferencias significativas entre las especies de diatomeas encontradas en los diferentes sitios. 
UNICIENCIA Vol. 31, No. 2, pp.99-109. Julio-diciembre, 2017.

ISSN Electrónico: 2215-3470

URL: www.revistas.una.ac.cr/uniciencia

DOI: http://dx.doi.org/10.15359/ru.31-2.7

Email: revistauniciencia@una.cr

\section{Conclusiones}

La presencia de especies como $N$. filiformes y N. palea y de N. crytotenella así como $N$. minima evidencia condiciones sanitarias deterioradas en las aguas del río.

Es necesaria más investigación en esta línea, para generar bases de datos de diatomeas de los ríos urbanos, con el fin de poder establecer conclusiones certeras de la calidad de agua y, sobre todo, estrategias de manejo de estos mismos en el país.

\section{Referencias}

Alonso, A. (2006). Valoración del efecto de la degradación ambiental sobre los macroinvertebrados bentónicos en la cabecera del río Henares. Ecosistemas 15(2),102-105.

Ballestero, M. (2003). Tárcoles River Basin Costa Rica. Background Paper. Recuperado de http:// siteresources.worldbank.org/INTSAREGTOPWATRES/Resources/CostaRica Tarcoles Background FINAL.pdf

Blanco, S., Bécares, E., Hernández, N., \& Ector, L. (2007). Evaluación en la calidad de agua en los ríos de la cuenca del Duero mediante índices diatomológicos. Ingeriería Civil 146, 139-143.

Fenoglio, S., Badino, G., \& Bona, F. (2002). Benthic macroinvertebrate comunities as indicators of river environment quality: an experience in Nicaragua. Revista. Biología Tropical, 50(3/4), 1125-1131.

Goulart, M. \& Callisto, M. (2003). Bioindicadores de qualidade de água como ferramenta em estudos de impacto ambiental. Revista da FAPAM, 2(1).

Kelly, M. (2000). Identification of common benthic diatoms in rivers. Field Studies 9, 583-700.

Michels, A. (1998). Use of diatoms (Bacillariophyceae) for water quality assessment in two tropical streams in Costa Rica. Revista BioIogía. Tropical, 46(Supl. 6), 143-152.

Miranda, O., Solano, V. y Balmaceda, G. (2010). II Taller: Construyendo acciones para la recuperación del río Pirro, Costa Rica. Universidad Nacional.

Naumoski, A. \& Mitreski, K. (2010). Classifying diatoms into trophic state index classes with novel classification algorithm. Procedia Environmental Sciences 2, 1124-1138. http://dx.doi.org/10.1016/j. proenv.2010.10.122

Penalta-Rodríguez, M. \& López-Rodríguez, M. C. (2007). Diatomeas y calidad del agua de los ríos del macizo central Gallego (Ourense, N.O. España) mediante la aplicación de índices diatomológicos. Limnetica, 26(2), 351-358.

Rice, E., Baird, R., Eaton, A \& Clesceri, L. (2012). Standard Methods for the Examination of Water and Wastewater (22 $2^{\text {th }}$ Ed.). Washington, USA.

Romero, M. (2008). Diagnóstico físico-ambiental de la microcuenca del río Pirro. Informe final de Proyecto. Escuela de Ciencias Geográficas, Universidad Nacional-Costa Rica.

Romero, M., Piedra L., Villalobos, R., Marín, R. y Núñez, F. (2011). Evaluación ecológica rápida de un ecosistema urbano: El caso de la microcuenca del río Pirro, Heredia, Costa Rica. Revista Geográfica América Central, 47(2), 41-70.

Sabater, S., Sabater, F. \& Tomas, X. (1987). Water quality and diatom communities in two catalan rivers. Water Research, 21(8), 901-911. http://dx.doi.org/10.1016/S0043-1354(87)80007-6.

Siqueiros-Beltrones, D., Argumedo-Hernández, U., Murillo-Jiménez, J., y Marmolejo-Rodríguez, A. (2014). Diversidad de diatomeas bentónicas marinas en un ambiente ligeramente enriquecido con elementos potencialmente tóxicos. Revista Mexicana de Biodiversidad 85, 1065-1085. http://dx.doi. org/10.7550/rmb.43748 
ISSN Electrónico: 2215-3470

DOI: http://dx.doi.org/10.15359/ru.31-2.7
UNICIENCIA Vol. 31, No. 2, pp. 99-109. Julio-diciembre, 2017. URL: www.revistas.una.ac.cr/uniciencia Email: revistauniciencia@una.cr

Silva-Benavides, M., Sili, C. \& Torzillom, G. (2008). Cyanoprocariota y microalgas (Chlorophyceae y Bacillariophyceae) bentónicas dominantes en ríos de Costa Rica. Revista Biología. Tropical 56(4), 221-235. Springer, M. (2010). Biomonitoreo acuático. Revista Biología Tropical, 58(supl), 4.

Werner, D. (1977). The Biology of Diatoms. University of California Press. Los Angeles, California.

Wang, J., Liu, X. D. \& Lu, J. (2012). Urban River Pollution Control and Remediation. Procedia Environmental Sciences 13, 1856-1862. http://dx.doi.org/10.1016/j.proenv.2012.01.179

\section{(9) $\odot \Theta \Theta$}

Evaluación breve de la presencia de diatomeas y su relación con algunos parámetros físico-químicos en el río Pirro, Heredia, Costa Rica (Eunice Flores-Stulzer y otros) por Revista Uniciencia se encuentra bajo una Licencia CreativeCommons Atribución-NoComercial-SinDerivadas 3.0 Unported. 